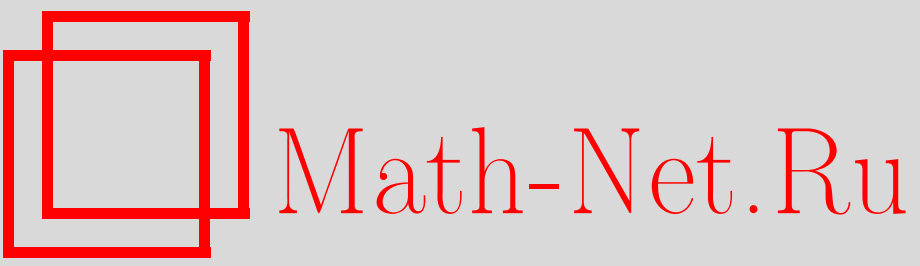

Е. В. Ферапонтов, Р. А. Шарипов, О законах сохранения первого порядка для систем уравнений гидродинамического типа, ТМФ, 1996, том 108, номер 1, 109 128

DOI: https://doi.org/10.4213/tmf1181

Использование Общероссийского математического портала Math-Net.Ru подразумевает, что вы прочитали и согласны с пользовательским соглашением http://www.mathnet.ru/rus/agreement

Параметры загрузки:

IP : 3.80 .253 .173

26 апреля 2023 г., 08:23:50 
ТЕОРЕТИЧЕСКАЯ

И МАТЕМАТИЧЕСКАЯ

ФИЗИКА

Том 108, № 1

июль, 1996

Е.В. Ферапонтов, Р. А. Шарипов

\section{О ЗАКОНАХ СОХРАНЕНИЯ ПЕРВОГО ПОРЯДКА ДЛЯ СИСТЕМ УРАВНЕНИЙ ГИДРОДИНАМИЧЕСКОГО ТИПА}

Рассмотрены квадратичные по производным законы сохранения первого порядка для систем уравнений гидродинамического типа. Выведены определяющие соотношения для плотностей таких законов сохранения, и найдена инвариантная относительно точечных замен переменных форма записи определяющих соотношений. Приведены примеры недиагонализуемых систем, допускающих квадратичные законы сохранения.

\section{1. ВВЕДЕНИЕ}

В данной работе рассматривается класс квазилинейных систем уравнений в частных производных, известный как класс уравнений гидродинамического типа:

$$
u_{t}^{i}=A_{j}^{i}(\mathbf{u}) u_{x}^{j}, \quad i=1, \ldots, n
$$

Этот класс уравнений выдерживает обратимые точечные преобразования вида

$$
\tilde{u}^{i}=\tilde{u}^{i}\left(u^{1}, \ldots, u^{n}\right),
$$

при которых компоненты матрицы $A_{j}^{i}(\mathbf{u})$ преобразуются как компоненты тензорного поля типа $(1,1)$ при замене локальных координат $u^{i}$ на $\tilde{u}^{i}$. Последнее обстоятельство позволяет привлекать различные дифференциально-геометрические методы к исследованию таких уравнений.

Система уравнений (1.1) называется диагонализуемой, если преобразованием (1.2) она приводится к диагональному виду

$$
u_{t}^{i}=\lambda_{i}(\mathbf{u}) u_{x}^{i}, \quad i=1, \ldots, n
$$

(суммирования по $i$ нет). Переменные $u^{i}$ в этом случае называются инвариантами Римана.

ОПРЕДЕЛЕНИЕ 1. Система уравнений (1.1) называется гидродинамически интегрируемой, если она обладает континуальным набором гидродинамических симметрий (или гидродинамических законов сохранения), который параметризуется $n$ произвольными функциями одной переменной. 
Напомним, что гидродинамический закон сохранения для системы уравнений (1.1) это интегральный функционал вида

$$
F=\int f(\mathbf{u}) d x
$$

производная по времени которого в силу этих уравнений равна нулю. Вопрос о гидродинамической интегрируемости уравнений (1.1) хорошо изучен для систем с матрицей обшего положения, собственные числа которой вешественны и попарно различны (см. обзоры $[1,2])$. Решение этого вопроса формулируется так.

ТЕОрема 1. Система уравнений (1.1) с матрицей общего положсения гидродинамически интегрируема тогда и только тогда, когда она диагонализуема и полугамильтонова.

Условие полугамильтоновости для диагональных систем (1.3) записывается в виде следующих соотношений:

$$
\partial_{i}\left(\frac{\partial_{j} \lambda_{k}}{\lambda_{j}-\lambda_{k}}\right)=\partial_{j}\left(\frac{\partial_{i} \lambda_{k}}{\lambda_{i}-\lambda_{k}}\right), \quad i \neq k \neq j
$$

Критерий диагонализуемости для уравнений (1.1) обсуждался в [3], а инвариантная форма записи соотношений (1.5) для недиагональных (но диагонализуемых) систем (1.1) была найдена в [4].

В данной работе мы рассматриваем законы сохранения первого порядка

$$
F=\int f\left(\mathbf{u}, \mathbf{u}_{x}\right) d x
$$

Отметим, что законы сохранения вида (1.6) для диагональных систем (1.3) исследовались в работах $[2]$ и $[5,6]$. Недиагональные (и недиагонализуемые) системы уравнений (1.1) с высшими законами сохранения в размерности $n=3$ исследовались в [7]. Цель данной работы - исследовать такие системы в произвольной размерности.

Оказалось, что для недиагонализуемых систем (более точно, для систем, не допускающих ни одного инварианта Римана) законы сохранения первого порядка с необходимостью являются квадратичными по $\mathbf{u}_{x}$,

$$
F=\int g_{i j}(\mathbf{u}) u_{x}^{i} u_{x}^{j} d x
$$

(см. раздел 2 ниже). Коэффициенты $g_{i j}$ (интерпретируемые как компоненты метрики) и матрица $A_{j}^{i}$ связаны соотношениями

$$
\begin{gathered}
A_{i j}=A_{j i} \\
\nabla_{k} A_{i j}+\nabla_{i} A_{j k}+\nabla_{j} A_{k i}=0 .
\end{gathered}
$$

Здесь $A_{i j}=g_{i s} A_{j}^{s}$, а $\nabla$ - ковариантное дифференцирование, порожденное метрикой $g_{i j}$. Соотношение (1.9) означает, что симметричный тензор $A_{i j}$ является тензором Киллинга для метрики $g_{i j}$, или же, что эквивалентно, выражение

$$
h=A_{i j}(\mathbf{u}) u_{x}^{i} u_{x}^{j}
$$


является квадратичным интегралом геодезических метрики $g_{i j}$ (см., например, [8]). Уравнение Киллинга (1.9) может быть получено на основе следуюшего соотношения, проверяемого непосредственным вычислением:

$$
\frac{\partial}{\partial t} g_{i j} u_{x}^{i} u_{x}^{j}=\frac{\partial}{\partial x} A_{i j} u_{x}^{i} u_{x}^{j}+\nabla_{k} A_{i j} u_{x}^{i} u_{x}^{j} u_{x}^{k}
$$

Из него видим, что функционал (1.7) является законом сохранения системы (1.1) в смысле определения из раздела 2 (см. ниже) тогда и только тогда, когда

$$
\nabla_{k} A_{i j} u_{x}^{i} u_{x}^{j} u_{x}^{k} \equiv 0
$$

Отсюда уравнения Киллинга получаются симметризацией по индексам $i, j, k$.

Имеется полезная конструкция (см. $[9,10])$, которая позволяет по всякому интегралу (1.7) строить запас точных решений исходной системы (1.1), сводя их отыскание к интегрированию конечномерной гамильтоновой системы, получаемой ограничением исходной системы на множество стационарных точек ее интеграла. В нашем случае эта конечномерная система есть не что иное, как уравнения геодезических метрики $g_{i j}$. Более точно, рассмотрим стандартное $2 n$-мерное симплектическое пространство с координатами $u^{i}, p_{i}$ и пару коммутирующих квадратичных гамильтонианов

$$
H_{1}=\frac{1}{2} g^{i j} p_{i} p_{j}, \quad H_{2}=\frac{1}{2} A^{i j} p_{i} p_{j}
$$

( $A^{i j}=g^{i k} A_{k}^{j}$; коммутация $H_{1}$ и $H_{2}$ следует из $\left.(1.8),(1.9)\right)$. Соответствующие уравнения движения имеют вид

$$
\begin{array}{ll}
u_{x}^{i}=\frac{\partial H_{1}}{\partial p_{i}}=g^{i j} p_{j}, & p_{i x}=-\frac{\partial H_{1}}{\partial u^{i}} \\
u_{t}^{i}=\frac{\partial H_{2}}{\partial p_{i}}=A^{i j} p_{j}, & p_{i t}=-\frac{\partial H_{2}}{\partial u^{i}}
\end{array}
$$

Здесь $x$ и $t$ - времена, отвечаюшие гамильтонианам $H_{1}$ и $H_{2}$. Исключая из $(1.10)$ и $(1.11)$ переменные $p_{i}$, мы приходим к уравнениям

$$
u_{t}^{i}=A_{j}^{i} u_{x}^{j}, \quad u_{x x}^{i}+\Gamma_{j k}^{i} u_{x}^{j} u_{x}^{k}=0 .
$$

где $\Gamma_{j k}^{i}$ - символы Кристоффеля афффинной связности, порожденной метрикой $g_{i j}$. Совместность переопределенной системы уравнений (1.12) является следствием (1.8), (1.9). Заметим, что второе из уравнений (1.12) есть не что иное, как уравнение стационарных точек интеграла $F: \frac{\delta F}{\delta u^{i}}=0$. Таким образом, всякий квадратичный интеграл (1.7) позволяет построить $2 n$-параметрическое семейство точных решений системы (1.1), сводя их отыскание к интегрированию пары коммутирующих гамильтоновых потоков (1.10) и (1.11), или же, что эквивалентно, к интегрированию переопределенной системы уравнений (1.12). Как легко видеть, всякое решение системы уравнений (1.12) "расслоено" на однопараметрическое семейство геодезических метрики $g_{i j}$. 
ЗАмЕЧАнИЕ. Представляет интерес исследовать совместность переопределенной системы уравнений (1.12) в самом общем случае, не накладывая изначально никаких ограничений на симметричную связность $\Gamma_{j k}^{i}$ (т.е. не считая, что эта связность является метрической и происходит из некоторого квадратичного интеграла исходной системы уравнений (1.1)). Используя тождество

$$
\frac{\partial}{\partial t}\left(u_{x x}^{i}+\Gamma_{j k}^{i} u_{x}^{j} u_{x}^{k}\right)=\left(\nabla_{k} \nabla_{l} A_{s}^{i}+R_{l k p}^{i} A_{s}^{p}\right) u_{x}^{k} u_{x}^{l} u_{x}^{s}
$$

которое выполняется в силу (1.12) (здесь $R_{l k p}^{i}-$ тензор кривизны связности $\Gamma_{j k}^{i}$ ), мы сразу же приходим к заключению, что совместность будет иметь место тогда и только тогда, когда

$$
\nabla_{k} \nabla_{l} A_{s}^{i}+R_{l k p}^{i} A_{s}^{p}+\cdots=0
$$

Многоточием обозначены члены, получаемые из выписанных симметризацией по индексам $k, l, s$ (отметим, что симметризация (1.13) по индексам $k, l, s$ совпадает с удвоенной циклической перестановкой по тем же индексам в силу тождеств Бьянки, которым удовлетворяет тензор кривизны любой симметричной связности). Всякая связность, удовлетворяющая соотношениям (1.13), позволяет построить $2 n$-параметрическое семейство решений исходной системы путем интегрирования соответствующих уравнений геодезических. Вопрос о том, какие системы (1.1) допускают связности (1.13) (и если допускают, то сколько), требует специального исследования.

Соотношения (1.8) и (1.9) накладывают жесткие ограничения не только на метрику $g_{i j}$, но и на сам аффинор $A_{j}^{i}$. Так, из (1.8) и (1.9) следует, что собственные числа $\lambda^{i}$ аффинора $A_{j}^{i}$ удовлетворяют условию

$$
L_{i} \lambda^{i}=0, \quad i=1, \ldots, n
$$

где $L_{i}$ - производная Ли вдоль правого собственного вектора $X_{i}$, отвечающего собственному значению $\lambda^{i}$. Системы (1.1), удовлетворяющие этому условию, носят название слабо нелинейных (linearly degenerate).

В качестве наиболее важных примеров недиагонализуемых систем, допускаюших квадратичные по производным интегралы, следует отметить матричное уравнение Хопpa

$$
U_{t}=\left(U^{2}\right)_{x}
$$

где $U$ - симметрическая матрица порядка $3 \times 3$ с дополнительной редукцией $\operatorname{tr} U^{i}=$ const, $i=1,2,3$, а также матричное уравнение

$$
U_{t}=\left(U U^{T} U\right)_{x}
$$

где $U$ - матрица порядка $2 \times 3$ с редукцией $\operatorname{tr}\left(U U^{T}\right)^{i}=\mathrm{const}, i=1,2$. Эти примеры подробно обсуждаются в разделе 4 . В обоих случаях имеется ровно два независимых интеграла, квадратичных по первым производным. 


\section{2. ЗАКОНЫ СОХРАНЕНИЯ ПЕРВОГО ПОРЯДКА}

Функционал (1.6) является законом сохранения или первым интегралом для уравнений (1.1), если его производная по $t$ в силу уравнений (1.1) равна нулю. Математически более точным (и более удобным) является следуюшее определение, которое эквивалентно сформулированному выше при достаточно разумных предположениях о поведении функций $u^{i}(x, t)$ при $x \rightarrow \pm \infty$.

ОПРЕДЕлЕниЕ 2. Функционал (1.6) является законом сохранения для уравнений (1.1), если найдется функция $h=h\left(\mathbf{u}, \mathbf{u}_{x}\right)$, такая что дифференциальная 1-форма $\omega=f d x+h d t$ замкнута для любого решения $\mathbf{u}(x, t)$ этих уравнений.

Замкнутость формы $\omega$ из определения 2 означает следующее:

$$
\frac{\partial f}{\partial t}=\frac{\partial h}{\partial x}
$$

Вычислим производные $\partial f / \partial t$ и $\partial h / \partial x$ из $(2.1)$ :

$$
\begin{gathered}
\frac{\partial f}{\partial t}=\sum_{i=1}^{n} \sum_{j=1}^{n}\left(\frac{\partial f}{\partial u^{i}} A_{j}^{i} u_{x}^{j}+\frac{\partial f}{\partial u_{x}^{i}} A_{j}^{i} u_{x x}^{j}+\sum_{k=1}^{n} \frac{\partial f}{\partial u_{x}^{i}} \frac{\partial A_{j}^{i}}{\partial u^{k}} u_{x}^{j} u_{x}^{k}\right), \\
\frac{\partial h}{\partial x}=\sum_{j=1}^{n}\left(\frac{\partial h}{\partial u^{j}} u_{x}^{j}+\frac{\partial h}{\partial u_{x}^{j}} u_{x x}^{j}\right) .
\end{gathered}
$$

Сравнивая (2.2) с (2.3), получаем следующие соотношения:

$$
\begin{gathered}
\frac{\partial h}{\partial u_{x}^{j}}=\sum_{i=1}^{n} \frac{\partial f}{\partial u_{x}^{i}} A_{j}^{i}, \\
\sum_{j=1}^{n} \frac{\partial h}{\partial u^{j}} u_{x}^{j}=\sum_{i=1}^{n} \sum_{j=1}^{n}\left(\frac{\partial f}{\partial u^{i}} A_{j}^{i} u_{x}^{j}+\sum_{k=1}^{n} \frac{\partial f}{\partial u_{x}^{i}} \frac{\partial A_{j}^{i}}{\partial u^{k}} u_{x}^{j} u_{x}^{k}\right) .
\end{gathered}
$$

Продифференцируем первое из соотношений (2.4) по $u_{x}^{k}$. В результате получим

$$
\frac{\partial^{2} h}{\partial u_{x}^{j} \partial u_{x}^{k}}=\sum_{i=1}^{n} g_{i k} A_{j}^{i}
$$

где $g_{i k}=\frac{\partial^{2} f}{\partial u_{x}^{i} \partial u_{x}^{k}}$. Теперь воспользуемся симметричностью смешанной производной в левой части (2.5) для получения уравнения, не содержашего функции $h$. Альтернируя по индексам $j$ и $k$, из $(2.5)$ находим

$$
\sum_{i=1}^{n} A_{j}^{i} g_{i k}=\sum_{i=1}^{n} g_{j i} A_{k}^{i}
$$

причем $g_{i j}=g_{j i}$. При точечных преобразованиях (1.2) величины $g_{i j}$ из (2.5) и (2.6) преобразуются как компоненты дважды ковариантного симметричного тензора. Однако зависимость этих величин от $\mathbf{u}_{x}$ не позволяет трактовать их как компоненты тензора 
в обычном понимании - это элемент расширенной алгебры тензорных полей. Идея рассмотрения таких нестандартных тензорных полей восходит к работам Финслера и Картана $[11,12]$. Применение этих объектов в теории ньютоновских динамических систем было найдено недавно в работах [13] и [14].

Пусть $\mathbf{X}_{1}, \ldots, \mathbf{X}_{n}$ - репер из собственных векторов матрицы скоростей $A_{j}^{i}$ и пусть $\mathbf{w}^{1}, \ldots, \mathbf{w}^{n}$ - сопряженный ему репер из ковекторов (1-форм). Выбор таких реперов неоднозначен, однако для матрицы $A_{j}^{i}(\mathbf{u})$ общего положения эта неоднозначность сводится к калибровочным преобразованиям

$$
\mathbf{X}_{i}(\mathbf{u}) \longrightarrow \varphi_{i}(\mathbf{u}) \mathbf{X}_{i}(\mathbf{u}), \quad \mathbf{w}^{i}(\mathbf{u}) \longrightarrow \varphi_{i}(\mathbf{u})^{-1} \mathbf{w}^{i}(\mathbf{u}) .
$$

Выбор реперов из (2.7) позволяет получить следующие разложения для единичного оператора $\mathbf{1}$ и для оператора $\mathbf{A}(\mathbf{u})$ :

$$
\mathbf{1}=\sum_{i=1}^{n} \mathbf{X}_{i} \otimes \mathbf{w}^{i}, \quad \mathbf{A}=\sum_{i=1}^{n} \lambda_{i} \mathbf{X}_{i} \otimes \mathbf{w}^{i}
$$

Разложим вектор $\mathbf{u}_{x}$ из аргумента функций $f$ и $h$ по этому реперу,

$$
\mathbf{u}_{x}=\sum_{i=1}^{n} y^{i} \mathbf{X}_{i}
$$

где $y^{i}=\mathbf{w}^{i} \cdot \mathbf{u}_{x}$. Тогда функции $f$ и $h$ можно считать функциями от коэффициентов такого разложения $f=f(\mathbf{u}, \mathbf{y})$ и $h=h(\mathbf{u}, \mathbf{y})$. Такие функции будем называть представлениями скалярных полей $f$ и $h$ в репере. Использование такого представления при учете соотношений (2.8) и (2.9) позволяет переписать первое из уравнений (2.4) в следуюшем виде:

$$
\frac{\partial h}{\partial y^{i}}=\lambda_{i}(\mathbf{u}) \frac{\partial f}{\partial y^{i}}
$$

Немедленным следствием (2.10) является следуюшее разделение переменных:

$$
f=\sum_{i=1}^{n} f_{i}\left(\mathbf{u}, y^{i}\right), \quad h=\sum_{i=1}^{n} \lambda_{i}(\mathbf{u}) f_{i}\left(\mathbf{u}, y^{i}\right) .
$$

Подставим представление $f$ и $h$ в форме (2.11) во второе уравнение (2.4). В результате получим

$$
\begin{gathered}
\sum_{i=1}^{n} \sum_{j=1}^{n}\left(\lambda_{i}-\lambda_{j}\right) y^{i} L_{i} f_{j}+\sum_{i=1}^{n} \sum_{j=1}^{n} y^{i}\left(\frac{\partial f_{j}}{\partial y^{j}} y^{j}-f_{j}\right) L_{i} \lambda_{j}= \\
=\frac{1}{2} \sum_{i=1}^{n} \sum_{j=1}^{n} \sum_{k=1}^{n}\left(\lambda_{i}-\lambda_{j}\right) c_{i j}^{k} \frac{\partial f_{k}}{\partial y^{k}} y^{i} y^{j}
\end{gathered}
$$

где через $L_{i}$ обозначены производные Ли вдоль собственных векторов матрицы скоростей $A_{j}^{i}$

$$
L_{i}=L_{\mathbf{x}_{i}}
$$


Структурные скаляры $c_{i j}^{k}(\mathbf{u})$ репера $\mathbf{X}_{1}, \ldots, \mathbf{X}_{n}$ из (2.12) определены посредством (2.13) из следуюших соотношений:

$$
L_{i} \mathbf{X}_{j}=\left[\mathbf{X}_{i}, \mathbf{X}_{j}\right]=\sum_{k=1}^{n} c_{i j}^{k} \mathbf{X}_{k}
$$

Выберем три попарно различных значения $i \neq j \neq k \neq i$ и вычислим смешанную производную (2.12) третьего порядка по $y^{i}, y^{j}$ и $y^{k}$. Левая часть этого соотношения при таком дифференцировании обрашается в нуль. Дифференцирование правой части дает

$$
\ddot{f}_{k} c_{i j}^{k}\left(\lambda_{i}-\lambda_{j}\right)+\ddot{f}_{i} c_{j k}^{i}\left(\lambda_{j}-\lambda_{k}\right)+\ddot{f}_{j} c_{k i}^{j}\left(\lambda_{k}-\lambda_{i}\right)=0
$$

где через $\ddot{f}_{k}$ обозначена вторая производная $f_{k}$ по $y^{k}$.

ОПРЕДЕЛЕНИЕ 3. Закон сохранения (1.6) для уравнений (1.1) называется законом сохранения с сушественно непостоянной сепарантой, если $\dddot{f}_{k} \not \equiv 0$ для всех $k=1, \ldots, n$.

Для законов сохранения с сушественно непостоянной сепарантой производные $\ddot{f}_{i}, \ddot{f}_{j}$ и $\ddot{f}_{k}$ линейно независимы над полем функций, не зависящих от $y^{1}, \ldots, y^{n}$. Немедленным следствием этого являются соотношения

$$
c_{i j}^{k}=0, \quad i \neq j \neq k \neq i
$$

Имеет место следуюшая хорошо известная

Лемма 1. Для того чтобъ линейный оператор $\mathbf{A}=\mathbf{A}(\mathbf{u})$ общего положсения был диагонализуем точечным преобразованием (1.2), необходимо и достаточно, чтобы выполнялись соотношения (2.16).

Применяя лемму 1 к нашему случаю, получаем следуюшую важную структурную теорему.

Теорема 2. Всякая система уравнений (1.1) с матрицей общего положения, обладающая законом сохранения (1.6) с существенно непостоянной сепарантой, диагонализуема.

Мы не будем исследовать диагонализуемый случай, ибо он достаточно подробно исследован в работах [5] и [6]. Противоположным является случай законов сохранения (1.6) с константной сепарантой.

ОПРЕДЕЛЕнИЕ 4. Закон сохранения (1.6) для уравнений (1.1) называется законом сохранения с константной сепарантой, если $\dddot{f}_{k} \equiv 0$ для всех $k=1, \ldots, n$.

Обший случай является промежуточным между двумя крайними случаями из определений 3 и 4 . Его рассмотрение есть предмет отдельной публикации. 


\section{3. ГЕОМЕТРИЯ ЗАКОНОВ СОХРАНЕНИЯ С КОНСТАНТНОЙ СЕПАРАНТОЙ}

Условие $\dddot{f}_{k} \equiv 0$, определяюшее закон сохранения с константной сепарантой, означает квадратичность $f_{k}$ по $y^{k}$. Величины $g_{i j}$ из $(2.5)$ в этом случае не зависят от $\mathbf{u}_{x}$, а сама плотность закона сохранения (1.6) может быть специализирована к виду

$$
f=\sum_{i=1}^{n} \sum_{j=1}^{n} g_{i j}(\mathbf{u}) u_{x}^{i} u_{x}^{j}+\sum_{i=1}^{n} M_{i}(\mathbf{u}) u_{x}^{i}+N(\mathbf{u})
$$

ОПРЕДЕЛЕниЕ 5. Закон сохранения с константной сепарантой (3.1) называется невырож денным, если невырожденна матрица $g_{i j}$, определяюшая в главном зависимость его плотности $f$ от $\mathbf{u}_{x}$.

В невырожденном случае матрица $g_{i j}$ задает риманову (или псевдориманову) метрику. В силу (2.6) операторнозначное поле $\mathbf{A}(\mathbf{u})$, задаваемое матрицей скоростей системы уравнений (1.1), состоит из симметрических операторов относительно метрики $g_{i j}$. Метрика $g_{i j}$ диагональна в репере из собственных векторов операторного поля $\mathbf{A}$, причем величины $\ddot{f}_{k}$ из $(2.15)$ - это в точности диагональные компоненты тензора $g_{i j}$ в таком репере: $\ddot{f}_{k}=g_{k k}=g\left(\mathbf{X}_{k}, \mathbf{X}_{k}\right)$. Сами же соотношения (2.15) для трех не совпадаюших значений индексов $i, j$ и $k$ можно переписать в виде

$$
g_{k k} c_{i j}^{k}\left(\lambda_{i}-\lambda_{j}\right)+g_{i i} c_{j k}^{i}\left(\lambda_{j}-\lambda_{k}\right)+g_{j j} c_{k i}^{j}\left(\lambda_{k}-\lambda_{i}\right)=0 .
$$

Соотношения (3.2) следует рассматривать как систему линейных уравнений на структурные скаляры $c_{i j}^{k}$, определенные согласно (2.14). Воспользуемся соотношениями $(2.12)$, подставив в них $f_{k}$ в форме, учитываюшей их квадратичную зависимость от $y^{k}$,

$$
f_{k}=g_{k k}\left(y^{k}\right)^{2}+M_{k} y^{k}+N_{k} .
$$

Суммирования по $k$ в (3.3) нет. Величины $M_{k}$ в (3.3) являются компонентами ковекторного поля М в репере $X_{1}, \ldots, X_{n}$. Выбор величин $N_{k}$ неоднозначен (они не являются компонентами ковектора). Единственное условие, которому должен удовлетворять выбор этих величин, выражается соотношениями

$$
\sum_{k=1}^{n} N_{k}=\left.f\right|_{\mathbf{y}=0}, \quad \sum_{k=1}^{n} \lambda_{k} N_{k}=\left.h\right|_{\mathbf{y}=0}
$$

Соотношения (3.4) вытекают из (2.11). Подстановка (3.3) в (2.12) приводит к соотношению, в которое входят слагаемые первой, второй и третьей степеней по у. Это позволяет разделить его на три соотношения. Первое имеет вид

$$
\lambda_{i} L_{i}\left(\sum_{j=1}^{n} N_{j}\right)=L_{i}\left(\sum_{j=1}^{n} \lambda_{j} N_{j}\right) .
$$

Сворачивая (3.5) по индексу $i$ с компонентами $X^{i}$ произвольного тензорного поля $\mathbf{X}$ в репере $\mathbf{X}_{1}, \ldots, \mathbf{X}_{n}$ и учитывая (3.4), получаем

$$
L_{\mathbf{A X}} f(\mathbf{u}, \mathbf{0})=L_{\mathbf{X}} h(\mathbf{u}, \mathbf{0}) .
$$


Мы можем исключить функцию $h(\mathbf{u}, \mathbf{0})$ после рассмотрения пары соотношений вида (3.6) с двумя различными векторными полями $\mathbf{X}$ и $\mathbf{Y}$. В качестве условия совместности получим

$$
\left(L_{\mathbf{X}} L_{\mathbf{A Y}}-L_{\mathbf{Y}} L_{\mathbf{A X}}-L_{\mathbf{A}[\mathbf{X}, \mathbf{Y}]}\right) f(\mathbf{u}, \mathbf{0})=0 .
$$

Согласно результатам [4] соотношение (3.7) есть в точности определяюшее соотношение для плотности гидродинамического закона сохранения (1.4). Это наблюдение позволяет сформулировать следующую теорему.

ТЕОРЕМА 3. Для всякого закона сохранения (1.6) с константной сепарантой имеется редукиия его плотности

$$
\tilde{f}(\mathbf{u})=f(\mathbf{u}, \mathbf{0})=\left.f\left(\mathbf{u}, \mathbf{u}_{x}\right)\right|_{\mathbf{u}_{x=0}},
$$

являющаяся плотностью гидродинамического закона сохранения (1.4) для той жсе системы уравнений (1.1).

Напомним, что теорема доказана нами для систем уравнений (1.1) с матрицей скоростей общего положения, собственные числа которой вешественны и попарно различны.

Второе соотношение, вытекаюшее из подстановки (3.3) в (2.12), имеет следуюший вид:

$$
L_{i} M_{j}-L_{j} M_{i}-\sum_{k=1}^{n} c_{i j}^{k} M_{k}=0 .
$$

После свертки (3.8) по индексам $i$ и $j$ с компонентами двух векторных полей $\mathbf{X}$ и $\mathbf{Y}$ в репере из собственных векторов матрицы скоростей $\mathbf{A}(\mathbf{u})$ получаем соотношение

$$
L_{\mathbf{X}}(\mathbf{Y} \cdot \mathbf{M})-L_{\mathbf{Y}}(\mathbf{X} \cdot \mathbf{M})-[\mathbf{X}, \mathbf{Y}] \cdot \mathbf{M}=0 .
$$

Это соотношение эквивалентно замкнутости 1-формы М, которой определяется линейная по $\mathbf{u}_{x}$ часть плотности закона сохранения (3.1). Это обстоятельство приводит к следуюшей теореме.

Теорема 4. Система уравнений (1.1) общего положсения не имеет нетривиальных законов сохранения первого порядка, линейных по $\mathbf{u}_{x}$, и линейная часть любого закона сохранения с невырожденной константной сепарантой может бъть отброшена.

Результаты, подобные теоремам 3 и 4, содержатся в [7] для уравнений (1.1) в размерности $n=3$. Из соотношения (3.1) и доказанных выше теорем 3 и 4 вытекает следуюшее утверж дение.

ТЕОрема 5. Всякий первый интеграл системы уравнений (1.1) общего положения, имеющий константную сепаранту, квадратичен по производным $\mathbf{u}_{x}$ и имеет вид (1.7).

Отметим, что всякое отклонение от условия константности сепаранты в законе сохранения первого порядка (1.6) влечет частичную диагонализуемость системы уравнений (1.1). Действительно, пусть одна из производных $\dddot{f}_{k}$ отлична от нуля, для определенности можно считать $\dddot{f}_{n} \neq 0$. Тогда из соотношения (2.15) получаем

$$
c_{i j}^{n}=0 \text { для всех } i \neq n \neq j .
$$


Значит, собственные векторы матрицы скоростей $\mathbf{X}_{1}, \ldots, \mathbf{X}_{n-1}$ порождают инволютивное распределение. Это означает, что точечным преобразованием (1.2) уравнения (1.1) приводятся к виду

$$
\left\{\begin{array}{l}
u_{t}^{i}=\sum_{j=1}^{n} A_{j}^{i}(\mathbf{u}) u_{x}^{j}, \quad i=1, \ldots, n-1, \\
u_{t}^{n}=A_{n}^{n}(\mathbf{u}) u_{x}^{n},
\end{array}\right.
$$

имеюшему один инвариант Римана $\lambda_{n}=A_{n}^{n}$. Полученное утверждение сформулируем в виде теоремы.

Теорема 6. Всякий первый интеграл системы уравнений (1.1) общего положения, не имеющей ни одного инварианта Римана, является интегралом с константной сепарантой. Он квадратичен по производным $\mathbf{u}_{x}$ и имеет вид (1.7).

Это утверждение было декларировано в разделе 1 . Вместе с предположением о невырожденности матришы $g_{i j}$ в (1.7) оно использовалось при выводе соотношений (1.8) и (1.9). При этом, по существу, была доказана следуюшая теорема.

ТеОРема 7. Метрика $g_{i j}$ определяет квадратичный по $\mathbf{u}_{x}$ закон сохранения для системы (1.1) тогда и только тогда, когда она диагонализуется в репере из собственных векторов матрицы скоростей и тензор $A_{i j}$ является тензором Киллинга.

Дальнейшее исследование законов сохранения первого порядка в случае существенно недиагонализуемых систем уравнений (1.1) основывается на соотношениях (1.8) и (1.9). Первое из них означает диагональность метрики $g_{i j}$ в репере из собственных векторов матрицы скоростей. Запись второго соотношения в этом репере несколько сложнее. Для вычисления ковариантных производных из (1.9) в репере $\mathbf{X}_{1}, \ldots, \mathbf{X}_{n}$ определим компоненты метрической связности в этом репере из следуюшего соотношения:

$$
\nabla \mathbf{x}_{i} \mathbf{X}_{j}=\sum_{k=1}^{n} \Gamma_{i j}^{k} \mathbf{X}_{k}
$$

Несмотря на симметричность связности Леви-Чивиты ее компоненты в репере $\Gamma_{i j}^{k}$ из (3.9) не являются симметричными по $i$ и $j$. Имеет место соотношение, связываюшее их со структурными скалярами репера,

$$
\Gamma_{i j}^{k}-\Gamma_{j i}^{k}=c_{i j}^{k}
$$

В силу (3.10) из условия согласованности метрики и связности для $\Gamma_{i j}^{k}$ можно вывести следуюшие явные формулы:

$$
\begin{aligned}
\Gamma_{i j}^{k}= & \frac{1}{2}\left(\frac{L_{i} g_{j j}}{g_{j j}} \delta_{j}^{k}+\frac{L_{j} g_{i i}}{g_{i i}} \delta_{i}^{k}-\frac{L_{k} g_{i i}}{g_{k k}} \delta_{j}^{i}\right)+ \\
& +\frac{1}{2} c_{i j}^{k}+\frac{1}{2} \frac{g_{j j}}{g_{k k}} c_{k i}^{j}+\frac{1}{2} \frac{g_{i i}}{g_{k k}} c_{k j}^{i} .
\end{aligned}
$$


При несовпадающих значениях индексов формула (3.11) принимает вид

$$
\Gamma_{i j}^{k}=\frac{1}{2} c_{i j}^{k}+\frac{1}{2} \frac{g_{j j}}{g_{k k}} c_{k i}^{j}+\frac{1}{2} \frac{g_{i i}}{g_{k k}} c_{k j}^{i}, \quad i \neq j \neq k \neq i
$$

При совпадении значений каких-либо двух индексов (3.11) дает

$$
\begin{aligned}
\Gamma_{i i}^{k} & =-\frac{1}{2} \frac{L_{k} g_{i i}}{g_{k k}}+\frac{g_{i i}}{g_{k k}} c_{k i}^{i}, \quad k \neq i, \\
\Gamma_{i j}^{i} & =\frac{1}{2} \frac{L_{j} g_{i i}}{g_{i i}}+c_{i j}^{i}, \\
\Gamma_{i j}^{j} & =\frac{1}{2} \frac{L_{i} g_{j j}}{g_{j j}} .
\end{aligned}
$$

В формулах (3.14) и (3.15) совпадение индексов $i=j$ возможно, тогда как в формуле (3.13) оно исключается.

Возврашаясь к формуле (1.9), вычислим компоненты ковариантной производной тензора Киллинга в репере $\mathbf{X}_{1}, \ldots, \mathbf{X}_{n}$. Они определяются формулой

$$
\nabla_{k} A_{i j}=L_{k} A_{i j}-\sum_{s=1}^{n} A_{s j} \Gamma_{k i}^{s}-\sum_{s=1}^{n} A_{i s} \Gamma_{k j}^{s} .
$$

Напомним, что матрицы $A_{i j}$ и $g_{i j}$ в репере $\mathbf{X}_{1}, \ldots, \mathbf{X}_{n}$ диагональны, т.е.

$$
A_{i j}=\lambda_{i} g_{i i} \delta_{j}^{i}, \quad g_{i j}=g_{i i} \delta_{j}^{i} .
$$

Учет соотношений (3.17) при подстановке (3.12) в формулу (3.16) для несовпадаюших значений индексов $i \neq j \neq k \neq i$ дает

$$
\nabla_{k} A_{i j}=\frac{\lambda_{i}-\lambda_{j}}{2}\left(g_{j j} c_{k i}^{j}+g_{i i} c_{j k}^{i}-g_{k k} c_{i j}^{k}\right) .
$$

Подстановка (3.18) в уравнение (1.9) приводит к соотношению, в точности совпадаюшемy с (3.2).

Противоположным по отношению к только что рассмотренному случаю является случай полного совпадения всех индексов $i=j=k$. В этом случае соотношение (3.16) после подстановки в уравнение (1.9) с учетом (3.15) дает

$$
L_{i} \lambda_{i}=0 .
$$

Соотношение (3.19) не содержит компонент метрики $g_{i j}$. Оно накладывает ограничение на выбор самой матрицы скоростей. Системы уравнений (1.1), удовлетворяющие этому ограничению, носят название слабо нелинейных.

Остается рассмотреть промежуточный случай $i \neq j=k$. В этом случае из соотношения (3.16) с учетом (3.13), (3.14) и (3.15) получаем

$$
\frac{L_{i} g_{j j}}{g_{j j}}=-\frac{L_{i} \lambda_{j}}{\lambda_{i}-\lambda_{j}}+2 c_{i j}^{j}, \quad i \neq j .
$$

Заметим, что формулы типа (3.2) и (3.20) были получены ранее в [15]. В целом вся совокупность соотношений (3.2), (3.19) и (3.20) эквивалентна уравнениям (1.9). Несмотря на то что уравнения Киллинга (1.9) имеют простой и инвариантньй дифференциально-геометрический смысл, соотношения (3.2), (3.19) и (3.20) несут гораздо большую информацию. В частности, они позволяют легко получить оценку сверху числа квадратичных по $\mathbf{u}_{x}$ интегралов для сушественно недиагонализуемых систем (1.1). Обозначим через $r$ ранг линейной (по $g_{i i}$ ) системы $(3.2)$, вычисленный в точке обшего положения. 
ТЕОрема 8. Существенно недиагонализуемая система (1.1) порядка $n \times n$ может иметь не более $n-r$ линейно независимых интегралов первого порядка, квадратичных по $\mathbf{u}_{x}$.

ДоказАТЕЛЬСтво. Рассмотрим коэффициент $g_{j j}$ при фиксированном значении индекса $j$. Уравнения (3.20) дают явные выражения для $L_{i} g_{j j}$ при $i \neq j$. Для нахождения $L_{j} g_{j j}$ мы поступим следуюшим образом. Выберем пару индексов $i \neq k$, отличных от $j$, таких что $c_{i k}^{j} \neq 0$, после чего применим оператор $L_{j}$ к соотношению (3.2). Подставляя $L_{j} g_{i i}$ и $L_{j} g_{k k}$ из соотношений (3.20), мы получим явное выражение для $L_{j} g_{j j}$. Таким образом, все производные от $g_{j j}$ оказались выраженными через сами коэффициенты $g_{j j}$, т.е. мы получили на $g_{j j}$ систему уравнений Пфаффа, общее решение которой зависит не более чем от $n$ произвольных постоянных. Учитывая, что соотношения (3.2) задают $r$ дополнительных линейных ограничений, мы приходим к утверждению теоремы.

СЛЕДСТВИЕ 1. Недиагонализуемая система порядка $3 \times 3($ а также $4 \times 4)$ может иметь не более двух интегралов, квадратичных по и $\mathbf{u}_{x}$.

В самом деле, для $n=3$ имеется ровно одно соотношение (3.2), причем оно не обращается тождественно в ноль в силу недиагонализуемости. Случай $n=4$ не многим сложнее предыдушего. Пусть (без ограничения обшности) коэффициент $c_{12}^{3} \neq 0$. Тогда соотношение (3.2), записанное для тройки индексов $(1,2,3)$, содержит лишь коэффициенты $g_{11}, g_{22}, g_{33}$. Пусть $c_{i j}^{4} \neq 0$ для некоторой пары $i \neq j \in\{1,2,3\}$. Тогда соотношение $(3.2)$, записанное для тройки индексов $(4, i, j)$, будет заведомо линейно независимо с первым. Таким образом, $r \geq 2$, что и доказывает следствие 2 для $n=4$.

Приводимые в следуюшем разделе примеры показывают, что в случаях $n=3$ и $n=4$ полученные выше оценки для числа квадратичных интегралов у недиагонализуемых систем являются точными.

\section{4. ПРИМЕРЫ. МАТРИЧНЫЕ УРАВНЕНИЯ ХОПФА}

ПримеР 1. Рассмотрим матричное дифференциальное уравнение вида

$$
U_{t}=\left(U^{2}\right)_{x},
$$

где $U$ - симметрическая матрица порядка $3 \times 3$ с дополнительной редукцией

$$
\operatorname{tr} U=\text { const }, \quad \operatorname{tr} U^{2}=\text { const, } \quad \operatorname{tr} U^{3}=\text { const }
$$

(легко проверяется, что редукция (4.2) совместима с уравнениями (4.1)). Представим $U$ в виде

$$
U=Q^{-1} R Q
$$

где $Q \in S O(3), R=\operatorname{diag}\left(R^{1}, R^{2}, R^{3}\right)$. Здесь $R^{i}=$ const в силу (4.2). Подстановка (4.3) в (4.1) приводит к уравнению

$$
\left[Q_{t} Q^{-1}, R\right]=\left[Q_{x} Q^{-1}, R^{2}\right]
$$

где [, ] обозначает коммутатор. Поскольку матрица $Q \in S O(3)$ зависит от трех параметров (например, трех углов Эйлера), уравнение (4.4) представляет собой систему гидродинамического типа порядка $3 \times 3$, которая, как несложно показать [16], является 
недиагонализуемой. Эта система имеет ровно два интеграла, квадратичных по первым производным,

$$
\begin{aligned}
\frac{\partial}{\partial t} \operatorname{tr}\left[\left(Q_{x} Q^{-1}\right)^{2}\right] & =\frac{\partial}{\partial x} \operatorname{tr}\left[2 R\left(Q_{x} Q^{-1}\right)^{2}\right] \\
\frac{\partial}{\partial t} \operatorname{tr}\left[R\left(Q_{x} Q^{-1}\right)^{2}\right] & =\frac{\partial}{\partial x} \operatorname{tr}\left[R^{2}\left(Q_{x} Q^{-1}\right)^{2}+\left(R Q_{x} Q^{-1}\right)^{2}\right]
\end{aligned}
$$

Приведенные формулы становятся более прозрачными, если в уравнениях (4.4) перейти от матрицы $Q$ к переменным $y^{1}, y^{2}, y^{3}$ по формуле

$$
Q_{x} Q^{-1}=\left(\begin{array}{ccc}
0 & y^{3} & -y^{2} \\
-y^{3} & 0 & y^{1} \\
y^{2} & -y^{1} & 0
\end{array}\right)
$$

(переход от $Q$ к $y^{i}$ есть не что иное, как дифференциальная подстановка первого порядка). Из (4.4) сразу же вытекает, что

$$
Q_{t} Q^{-1}=\left(\begin{array}{ccc}
0 & y^{3} \lambda^{3} & -y^{2} \lambda^{2} \\
-y^{3} \lambda^{3} & 0 & y^{1} \lambda^{1} \\
y^{2} \lambda^{2} & -y^{1} \lambda^{1} & 0
\end{array}\right)
$$

где $\lambda^{1}=R^{2}+R^{3}, \lambda^{2}=R^{1}+R^{3}, \lambda^{3}=R^{1}+R^{2}$. Из (4.6) и (4.7) следует, что переменные $y^{1}, y^{2}, y^{3}$ удовлетворяют системе трех волн

$$
\begin{aligned}
& y_{t}^{1}=\lambda^{1} y_{x}^{1}+\left(\lambda^{3}-\lambda^{2}\right) y^{2} y^{3}, \\
& y_{t}^{2}=\lambda^{2} y_{x}^{2}+\left(\lambda^{1}-\lambda^{3}\right) y^{1} y^{3}, \\
& y_{t}^{3}=\lambda^{3} y_{x}^{3}+\left(\lambda^{2}-\lambda^{1}\right) y^{1} y^{2} .
\end{aligned}
$$

При этом интегралы (4.5) переходят в известные интегралы системы трех волн:

$$
\begin{gathered}
\frac{\partial}{\partial t}\left(y^{1^{2}}+y^{2^{2}}+y^{3^{2}}\right)=\frac{\partial}{\partial x}\left(\lambda^{1} y^{1^{2}}+\lambda^{2} y^{2^{2}}+\lambda^{3} y^{3^{2}}\right) \\
\frac{\partial}{\partial t}\left(\lambda^{1} y^{1^{2}}+\lambda^{2} y^{2^{2}}+\lambda^{3} y^{3^{2}}\right)=\frac{\partial}{\partial x}\left(\lambda^{1^{2}} y^{1^{2}}+\lambda^{2^{2}} y^{2^{2}}+\lambda^{3^{2}} y^{3^{2}}\right)
\end{gathered}
$$

Другие примеры недиагонализуемых систем, допускающих пару квадратичных интегралов, могут быть получены следуюшей простой конструкцией. Пусть $A(u) d t+$ $B(u) d x$ и $M(u) d t+N(u) d x$ - пара интегралов гидродинамического типа исходной системы (1.1). Переходя в уравнениях (1.1) от $x, t$ к новым независимым переменным $X, T$ по формулам

$$
d X=A d t+B d x, \quad d T=M d t+N d x
$$

мы придем к системе

$$
u_{T}^{i}=\widetilde{A}_{j}^{i}(u) u_{X}^{j}
$$

где новая матрица $\widetilde{A}_{j}^{i}$ связана с $A_{j}^{i}$ следующей формулой:

$$
\widetilde{A}_{j}^{i}=\left(A_{j}^{i} B-\delta_{j}^{i} A\right)\left(\delta_{j}^{i} M-A_{j}^{i} N\right)^{-1} .
$$


Преобразования типа (4.8) носят название преобразований "по решению" (reciprocal transformations), поскольку переменные $X, T$ явно зависят от решения $\mathbf{u}(x, t)$ системы (1.1). Легко видеть, что квадратичные по $\mathbf{u}_{x}$ интегралы исходной системы (если таковые имеются) переходят в квадратичные по $\mathbf{u}_{X}$ интегралы преобразованной системы по несложным формулам, вывод которых мы оставляем читателю. Таким образом, выбирая в качестве исходной некоторую систему, имеюшую квадратичные по $\mathbf{u}_{x}$ интегралы (например, систему (4.4), имеющую пару квадратичных интегралов) и применяя произвольные преобразования по решению, мы получим широкий запас примеров, которые также будут допускать квадратичные интегралы.

Подчеркнем, что система (4.4) имеет ровно 5 интегралов гидродинамического типа [16]. В самом деле, консервативная запись (4.1) дает 6 законов сохранения (напомним, что матрица $U$ является симметрической). Линейное соотношение $\operatorname{tr} U=$ const уменьшает их число на единицу (оставшиеся нелинейные соотношения (4.2) не приводят к дальнейшему уменьшению размерности пространства гидродинамических интегралов).

Следует отметить, что все известные на настоящий момент недиагонализуемые системы (1.1) порядка $3 \times 3$, допускаюшие пару интегралов, квадратичных по $\mathbf{u}_{x}$, сводятся к (4.4) преобразованиями по решению.

Аналогичным образом исследуется и обший случай матричного уравнения Хопфа

$$
U_{t}=\left(U^{2}\right)_{x}
$$

где $U$ - матрица порядка $n \times n$ с дополнительной редукцией

$$
\operatorname{tr} U^{k}=\text { const, } \quad k=1, \ldots, n \text {. }
$$

Это матричное уравнение представляет собой недиагонализуемую систему гидродинамического типа порядка $N \times N$, где $N=n(n-1) / 2$. Можно показать, что она имеет ровно $n-1$ интегралов, квадратичных по производным, а также ровно $n(n+1) / 2-1$ интегралов гидродинамического типа. Так, для $n=4$ плотности законов сохранения, квадратичные по производным, имеют вид

$$
\operatorname{tr}\left[\left(Q_{x} Q^{-1}\right)^{2}\right], \quad \operatorname{tr}\left[R\left(Q_{x} Q^{-1}\right)^{2}\right], \quad \operatorname{tr}\left[R^{2}\left(Q_{x} Q^{-1}\right)^{2}+\frac{1}{2}\left(R Q_{x} Q^{-1}\right)^{2}\right] .
$$

Здесь $Q \in S O(4)$ и $R=\operatorname{diag}\left(R^{1}, R^{2}, R^{3}, R^{4}\right)$ определяются из представления $U=$ $Q^{-1} R Q$. (При $n=3$ третья плотность является линейной комбинацией первых двух.)

ПримеР 2. Рассмотрим матричное дифференциальное уравнение вида

$$
U_{t}=\left(U U^{T} U\right)_{x}
$$

где $U$ - матрица размера $2 \times 3$ с дополнительной редукцией

$$
\operatorname{tr} U U^{T}=\text { const }, \quad \operatorname{tr}\left(U U^{T}\right)^{2}=\text { const }
$$

( $U^{T}$ обозначает транспонированную матрицу). Легко проверяется, что редукция (4.10) совместима с уравнениями (4.9). Представим $U$ в виде

$$
U=P^{-1} R Q
$$


где $P \in S O(2), Q \in S O(3)$, а $R$ - матрица размера $2 \times 3$ следуюшего специального вида:

$$
R=\left(\begin{array}{ccc}
\alpha & 0 & 0 \\
0 & \beta & 0
\end{array}\right)
$$

Здесь $\alpha=$ const, $\beta=$ const в силу (4.10). Подстановка (4.11) в (4.9) приводит к уравнению

$$
R Q_{t} Q^{-1}-P_{t} P^{-1} R=\widetilde{R} Q_{x} Q^{-1}-P_{x} P^{-1} \widetilde{R},
$$

где $\widetilde{R}=R R^{T} R$. Уравнение (4.12) представляет собой систему гидродинамического типа порядка $4 \times 4$ относительно коэффициентов матриц $P, Q$, которая, как несложно показать [17], является недиагонализуемой. Эта система имеет ровно два интеграла, квадратичных по первым производным, плотности которых имеют вид

$$
\begin{gathered}
\operatorname{tr}\left[\left(Q_{x} Q^{-1}\right)^{2}+\left(P_{x} P^{-1}\right)^{2}\right], \\
\operatorname{tr}\left[R^{T} R\left(Q_{x} Q^{-1}\right)^{2}+R R^{T}\left(P_{x} P^{-1}\right)^{2}+R Q_{x} Q^{-1} R^{T} P_{x} P^{-1}\right] .
\end{gathered}
$$

Приведенные формулы становятся более прозрачньми, если в (4.12) перейти от матриц $Q, P$ к переменным $y^{1}, y^{2}, y^{3}, y^{4}$ по формуле

$$
Q_{x} Q^{-1}=\left(\begin{array}{ccc}
0 & y^{3}+y^{4} & -y^{2} \\
-y^{3}-y^{4} & 0 & y^{1} \\
y^{2} & -y^{1} & 0
\end{array}\right), \quad P_{x} P^{-1}=\left(\begin{array}{cc}
0 & y^{3}-y^{4} \\
y^{4}-y^{3} & 0
\end{array}\right) .
$$

Из (4.12) сразу же вытекает, что

$$
\begin{aligned}
& Q_{t} Q^{-1}=\left(\begin{array}{ccc}
0 & y^{3} \lambda^{3}+y^{4} \lambda^{4} & -y^{2} \lambda^{2} \\
-y^{3} \lambda^{3}-y^{4} \lambda^{4} & 0 & y^{1} \lambda^{1} \\
y^{2} \lambda^{2} & -y^{1} \lambda^{1} & 0
\end{array}\right), \\
& P_{t} P^{-1}=\left(\begin{array}{cc}
0 & y^{3} \lambda^{3}-y^{4} \lambda^{4} \\
y^{4} \lambda^{4}-y^{3} \lambda^{3} & 0
\end{array}\right),
\end{aligned}
$$

где $\lambda^{1}=\beta^{2}, \lambda^{2}=\alpha^{2}, \lambda^{3}=\alpha^{2}+\alpha \beta+\beta^{2}, \lambda^{4}=\alpha^{2}-\alpha \beta+\beta^{2}$. Из (4.14) и (4.15) следует, что переменные $y^{1}, y^{2}, y^{3}, y^{4}$ удовлетворяют системе четырех волн

$$
\begin{aligned}
y_{t}^{1} & =\lambda^{1} y_{x}^{1}+\left(\lambda^{3}-\lambda^{2}\right) y^{2} y^{3}+\left(\lambda^{4}-\lambda^{2}\right) y^{2} y^{4}, \\
y_{t}^{2} & =\lambda^{2} y_{x}^{2}+\left(\lambda^{1}-\lambda^{3}\right) y^{1} y^{3}+\left(\lambda^{1}-\lambda^{4}\right) y^{1} y^{4}, \\
y_{t}^{3} & =\lambda^{3} y_{x}^{3}+\frac{1}{2}\left(\lambda^{2}-\lambda^{1}\right) y^{1} y^{2}, \\
y_{t}^{4} & =\lambda^{4} y_{x}^{4}+\frac{1}{2}\left(\lambda^{2}-\lambda^{1}\right) y^{1} y^{2} .
\end{aligned}
$$

В нашем примере двойное отношение четверки скоростей $\lambda^{i}$ равно -1 :

$$
\frac{\left(\lambda^{1}-\lambda^{4}\right)\left(\lambda^{2}-\lambda^{3}\right)}{\left(\lambda^{1}-\lambda^{3}\right)\left(\lambda^{2}-\lambda^{4}\right)}=-1
$$

При выполнении условия (4.17) система (4.16) является интегрируемой методом обратной задачи (см. $[17 ; 18$, с. 216$])$. По-видимому, $(4.17)$ является также и необходимым 
условием интегрируемости. Тем не менее при любых $\lambda^{i}$ система (4.16) имеет пару квадратичных интегралов

$$
\begin{gathered}
\frac{\partial}{\partial t}\left(y^{1^{2}}+y^{2^{2}}+2 y^{3^{2}}+2 y^{4^{2}}\right)=\frac{\partial}{\partial x}\left(\lambda^{1} y^{1^{2}}+\lambda^{2} y^{2^{2}}+2 \lambda^{3} y^{3^{2}}+2 \lambda^{4} y^{4^{2}}\right) \\
\frac{\partial}{\partial t}\left(\lambda^{1} y^{1^{2}}+\lambda^{2} y^{2^{2}}+2 \lambda^{3} y^{3^{2}}+2 \lambda^{4} y^{4^{2}}\right)=\frac{\partial}{\partial x}\left(\lambda^{1^{2}} y^{1^{2}}+\lambda^{2^{2}} y^{2^{2}}+2 \lambda^{3^{2}} y^{3^{2}}+2 \lambda^{4^{2}} y^{4^{2}}\right)
\end{gathered}
$$

плотности которых в точности отвечают плотностям (4.13).

Другие примеры недиагонализуемых систем порядка $4 \times 4$, допускающих пару интегралов, квадратичных по первым производным, могут быть получены применением преобразований по решению к системе (4.12) (отметим, что система (4.12) имеет ровно 6 интегралов гидродинамического типа).

Подчеркнем, что в обоих рассмотренных вьше примерах имеется бесконечное число высших интегралов (по два интеграла в каждом четном порядке по производным; при этом интегралы, квадратичные по первым производным, имеют второй порядок).

\section{5. ПРИЛОЖЕНИЕ. ИНВАРИАНТНАЯ ФОРМА ЗАПИСИ СООТНОШЕНИЙ $(3.2),(3.19),(3.20)$}

Соотношение (3.19), выведенное вьше прямым вычислением в репере из собственных векторов матришы $\mathbf{A}$, ввиду тензорного характера входяших в него величин остается верным в любых локальных координатах. При заданных матрицах $A_{j}^{i}$ и $g_{i j}$ его проверка не составляет труда. Однако оно плохо подходит для нахождения метрики $g_{i j}$ по заданной матрице скоростей. Поскольку оно содержит в себе три условия (3.2), (3.19), (3.20), естественно поставить вопрос об отдельном преобразовании соотношений (3.2), (3.19) и (3.20) в произвольную систему локальных координат $u^{1}, \ldots, u^{n}$.

Инвариантная форма записи условия слабой нелинейности (3.19) известна (см. [7] и [16]). Оно дается в терминах коэффишиентов характеристического полинома матришы скоростей. Пусть

$$
\mathrm{P}(\lambda)=\operatorname{det}(\lambda-\mathbf{A})=\lambda^{n}+\sum_{s=0}^{n-1} P_{s}(\mathbf{u}) \lambda^{s}
$$

и пусть $A^{(s)}{ }_{j}^{i}-s$-я степень матрицы скоростей $\mathbf{A}$. Тогда условие слабой нелинейности (3.19) эквивалентно следуюшим соотношениям:

$$
\sum_{s=0}^{n-1} \sum_{i=1}^{n} \frac{\partial P_{s}(\mathbf{u})}{\partial u^{i}} A_{j}^{(s)}{ }_{j}^{i}=0, \quad j=1, \ldots, n .
$$

Для записи соотношений (3.2) в произвольных локальных координатах воспользуемся тензором Хаантьеса Н. Напомним, что это тензорное поле типа $(1,2)$, которое строится по матрице $\mathbf{A}$. Предварительным этапом при этом является построение тензора Нейенхейса $\mathbf{N}$. Тензоры $\mathbf{N}$ и $\mathbf{H}$ могут быть определены формулами

$$
\begin{aligned}
& \mathbf{N}(\mathbf{X}, \mathbf{Y})=[\mathbf{A X}, \mathbf{A} \mathbf{Y}]+\mathbf{A}^{2}[\mathbf{X}, \mathbf{Y}]-\mathbf{A}[\mathbf{X}, \mathbf{A} \mathbf{Y}]-\mathbf{A}[\mathbf{A X}, \mathbf{Y}] \\
& \mathbf{H}(\mathbf{X}, \mathbf{Y})=\mathbf{N}(\mathbf{A X}, \mathbf{A Y})+\mathbf{A}^{2} \mathbf{N}(\mathbf{X}, \mathbf{Y})-\mathbf{A} \mathbf{N}(\mathbf{X}, \mathbf{A Y})-\mathbf{A N}(\mathbf{A X}, \mathbf{Y}),
\end{aligned}
$$


где $\mathbf{X}$ и $\mathbf{Y}$ - два произвольных векторных поля. Компоненты тензоров $\mathbf{N}$ и $\mathbf{H}$ вычисляются так:

$$
\begin{aligned}
N_{i j}^{k} & =\sum_{s=1}^{n}\left(A_{i}^{s} \partial_{s} A_{j}^{k}-A_{j}^{s} \partial_{s} A_{i}^{k}+A_{s}^{k} \partial_{j} A_{i}^{s}-A_{s}^{k} \partial_{i} A_{j}^{s}\right), \\
H_{i j}^{k} & =\sum_{s=1}^{n} \sum_{r=1}^{n}\left(A_{s}^{k} A_{r}^{s} N_{i j}^{r}-A_{s}^{k} N_{r j}^{s} A_{i}^{r}-A_{s}^{k} N_{i r}^{s} A_{j}^{r}+N_{s r}^{k} A_{i}^{s} A_{j}^{r}\right) .
\end{aligned}
$$

В репере из собственных векторов матрицы $\mathbf{A}$ тензор Хаантьеса выглядит чрезвычайно просто. Замечательное обстоятельство состоит в том, что его компоненты пропорциональны структурным скалярам репера:

$$
\mathbf{H}\left(\mathbf{X}_{i}, \mathbf{X}_{j}\right)=\sum_{k=1}^{n}\left(\lambda_{i}-\lambda_{k}\right)^{2}\left(\lambda_{j}-\lambda_{k}\right)^{2} c_{i j}^{k} \mathbf{X}_{k}
$$

Сравнивая (5.3) с (3.2), замечаем, что для записи (3.2) в тензорной форме необходимо ввести тензор $\mathbf{W}$, такой что

$$
\mathbf{W}\left(\mathbf{X}_{i}, \mathbf{X}_{j}\right)=\left(\lambda_{i}-\lambda_{j}\right)^{2} \mathbf{H}\left(\mathbf{X}_{i}, \mathbf{X}_{j}\right)
$$

Этот тензор можно определить инвариантным образом, не привязываясь к собственным векторам матрицы $\mathbf{A}$ :

$$
\mathbf{W}(\mathbf{X}, \mathbf{Y})=\mathbf{H}\left(\mathbf{A}^{2} \mathbf{X}, \mathbf{Y}\right)-2 \mathbf{H}(\mathbf{A X}, \mathbf{A Y})+\mathbf{H}\left(\mathbf{X}, \mathbf{A}^{2} \mathbf{Y}\right)
$$

Соотношениям (5.4) в координатах соответствуют следуюшие формулы для компонент тензора $\mathbf{W}$ :

$$
\mathbf{W}_{i j}^{k}=\sum_{p=1}^{n} \sum_{q=1}^{n}\left(A_{i}^{p} A_{p}^{q} H_{q j}^{k}-2 A_{i}^{p} A_{j}^{q} H_{q p}^{k}+A_{j}^{p} A_{p}^{q} H_{i q}^{k}\right) .
$$

Соотношение (3.2) теперь может быть переписано в следуюшей инвариантной форме:

$$
g(\mathbf{W}(\mathbf{A X}, \mathbf{Y})-\mathbf{W}(\mathbf{X}, \mathbf{A Y}), \mathbf{Z})+\cdots=0
$$

где $\mathbf{X}, \mathbf{Y}$ и $\mathbf{Z}$ - три произвольных векторных поля. Многоточием в (5.5) обозначены слагаемые, получаемые циклической перестановкой $\mathbf{X}, \mathbf{Y}$ и $\mathbf{Z}$. В локальных координатах (5.5) принимает вид

$$
\sum_{s=1}^{n} \sum_{q=1}^{n}\left(W_{q j}^{s} A_{i}^{q}-W_{i q}^{s} A_{j}^{q}\right) g_{s k}+\cdots=0
$$

недостаюшие слагаемые в (5.6) получаются циклической перестановкой индексов $i, j, k$. 
Запись уравнений (3.10) в произвольных локальных координатах требует значительно больших усилий. С репером собственных векторов матришы скоростей $\mathbf{A}(\mathbf{u})$ свяжем следующие величины:

$$
\begin{aligned}
& \bar{\Gamma}_{i j}^{k}=0, \quad i \neq j, j \neq k, k \neq i, \\
& \bar{\Gamma}_{i j}^{i}=0, \quad \bar{\Gamma}_{i i}^{j}=0, \quad i \neq j, \\
& \bar{\Gamma}_{i j}^{j}=c_{i j}^{j}-\frac{1}{2} \frac{L_{i} \lambda_{j}}{\lambda_{i}-\lambda_{j}}, \quad i \neq j .
\end{aligned}
$$

Величины $\bar{\Gamma}_{j k}^{i}$ из (5.7) преобразуются как компоненты некоторой связности при калибровочных преобразованиях репера (2.7). Величины $\bar{\Gamma}_{i i}^{i}$, не определенные в $(5.7)$, выберем произвольным образом, например, положив их равными нулю при некотором фиксированном выборе калибровки репера $\mathbf{X}_{1}, \ldots, \mathbf{X}_{n}$. В результате этого мы построим связность, определяемую только матрицей скоростей $\mathbf{A}(\mathbf{u})$, хотя и не совсем однозначно. Нетрудно проверить, что соотношение (3.10) может быть переписано в терминах ковариантных производных, определяемых этой связностью:

$$
\bar{\nabla}_{\mathbf{X}} g(\mathbf{Y}, \mathbf{Z})=\bar{\nabla}_{\mathbf{Y}} g(\mathbf{X}, \mathbf{Z}) .
$$

Остается лишь вопрос об инвариантном (не используюшем реперы $\mathbf{X}_{1}, \ldots, \mathbf{X}_{n}$ ) определении ковариантных производных из (5.8). В качестве первого этапа при этом определим тензор $\mathbf{C}$ типа $(1,2)$, задав его следуюшей формулой:

$$
\mathbf{C}\left(\mathbf{X}_{i}, \mathbf{X}_{j}\right)=\sum_{\substack{k \neq i \\ k \neq j}}^{n} c_{i j}^{k} \mathbf{X}_{k}
$$

Тензор $\mathbf{C}$ из (5.9) связан с тензором Хаантьеса (5.2) через промежуточный тензор $\mathbf{U}$

$$
\begin{aligned}
& \mathbf{U}(\mathbf{X}, \mathbf{Y})=\mathbf{C}(\mathbf{A X}, \mathbf{A Y})+\mathbf{A}^{2} \mathbf{C}(\mathbf{X}, \mathbf{Y})-\mathbf{A C}(\mathbf{X}, \mathbf{A Y})-\mathbf{A C}(\mathbf{A X}, \mathbf{Y}) \\
& \mathbf{H}(\mathbf{X}, \mathbf{Y})=\mathbf{U}(\mathbf{A X}, \mathbf{A Y})+\mathbf{A}^{2} \mathbf{U}(\mathbf{X}, \mathbf{Y})-\mathbf{A} \mathbf{U}(\mathbf{X}, \mathbf{A Y})-\mathbf{A} \mathbf{U}(\mathbf{A X}, \mathbf{Y}) .
\end{aligned}
$$

Соотношения (5.10) удается обратить, т.е. выразить тензор $\mathbf{C}$ через тензор Хаантьеса. Для осушествления такого обрашения рассмотрим полином двух переменных $x$ и $y$ следующего вида:

$$
Q(x, y)=\sum_{1 \leq i \neq k \leq n}\left(\frac{1}{\left(\lambda_{i}-\lambda_{k}\right)^{2}} \prod_{r \neq i}^{n} \frac{x-\lambda_{r}}{\lambda_{i}-\lambda_{r}} \prod_{s \neq k}^{n} \frac{y-\lambda_{s}}{\lambda_{k}-\lambda_{s}}\right) .
$$

Степень полинома (5.11) по каждой из переменных $x$ и $y$ равна $n-1$. Поэтому

$$
Q(x, y)=\sum_{p=0}^{n-1} \sum_{q=0}^{n-1} Q_{p q}\left(\lambda_{1}, \ldots, \lambda_{n}\right) x^{p} y^{q} .
$$

Коэффициенты полинома $Q(x, y)$ в $(5.12)$ являются симметрическими рациональными функциями собственных чисел матрищы скоростей А. Поэтому они рациональным образом выражаются через коэффициенты ее характеристического полинома. В силу сказанного вычисление полинома (5.11) по матрице скоростей, не будучи явным, является 
все же алгоритмически эффективным. Формула обрашения для (5.10) может быть записана так:

$$
\mathbf{C}(\mathbf{X}, \mathbf{Y})=\sum_{p=0}^{n-1} \sum_{q=0}^{n-1} \sum_{r=0}^{n-1} \sum_{s=0}^{n-1} Q_{p q} Q_{r s} \mathbf{A}^{q+s} \mathbf{H}\left(\mathbf{A}^{p} \mathbf{X}, \mathbf{A}^{r} \mathbf{Y}\right)
$$

В силу (5.10) и (5.13) компоненты тензоров $\mathbf{C}$ и $\mathbf{U}$ эффективно вычислимы по матрице скоростей в любых локальных координатах. Для тензора Нейенхейса имеется разложение $\mathbf{N}=\widetilde{\mathbf{N}}+\mathbf{U}$, причем

$$
\tilde{\mathbf{N}}\left(\mathbf{X}_{i}, \mathbf{X}_{j}\right)=\left(\lambda_{i}-\lambda_{j}\right)\left(\mathbf{X}_{i} L_{j} \lambda_{i}+\mathbf{X}_{j} L_{i} \lambda_{j}\right) .
$$

Компоненты тензора $\widetilde{\mathbf{N}}$ также эффективно вычислимы в любых локальных координатах. Назовем его главной частью тензора Нейенхейса. Используя коэффициенты полинома $Q(x, y)$ из (5.11) и тензор $\widetilde{\mathbf{N}}$ из (5.14), построим еше один тензор $\mathbf{D}$ по следуюшей формуле:

$$
\mathbf{D}(\mathbf{X}, \mathbf{Y})=\sum_{p=0}^{n-1} \sum_{q=0}^{n-1} \widetilde{\mathbf{N}}\left(\mathbf{A}^{p} \mathbf{X}, \mathbf{A}^{q} \mathbf{Y}\right)
$$

Тензор $\mathbf{D}$ входит в инвариантные определяюшие соотношения для связности $\bar{\nabla}$, которые имеют вид

$$
\begin{aligned}
& \bar{\nabla}_{\mathbf{X}} \mathbf{Y}-\bar{\nabla}_{\mathbf{Y}} \mathbf{X}=[\mathbf{X}, \mathbf{Y}]-\mathbf{C}(\mathbf{X}, \mathbf{Y})-\frac{1}{2} \mathbf{D}(\mathbf{X}, \mathbf{Y}) \\
& \bar{\nabla}_{\mathbf{X}} \mathbf{A} \mathbf{Y}+\bar{\nabla}_{\mathbf{Y}} \mathbf{A X}=\mathbf{D}(\mathbf{A X}, \mathbf{Y})-\mathbf{D}(\mathbf{X}, \mathbf{A} \mathbf{Y}) .
\end{aligned}
$$

Соотношения (5.16) определяют связность $\bar{\nabla}$ с той же степенью произвола, что и соотношения (5.7), задаюшие компоненты этой связности в репере из собственных векторов матрицы скоростей. Резюмируя полученные результаты, переформулируем теорему 5 в следуюшем виде.

Теорема 9. Метрика $g_{i j}$ определяет квадратичный по $\mathbf{u}_{x}$ закон сохранения для системы уравнений (1.1) тогда и только тогда, когда она диагонализуется в репере из собственных векторов матрицы скоростей и имеют место соотношения (5.1), (5.5) и (5.8).

Отметим, что соотношения (5.8) представляют собой переопределенную систему уравнений на метрику $g_{i j}$, условия совместности которых аналогичны условиям полугамильтоновости в диагонализуемом случае. Вывод и анализ этих условий совместности требует, однако, отдельного рассмотрения.

Работа выполнена при финансовой поддержке фонда Сороса (проекты № RK4000, RKR000) и Российского фонда фундаментальных исследований (проекты № 93-01-00273, 93-011-168). 


\section{Список литературы}

[1] Б.А.Дубровин, С.П.Новиков // УМН. 1989. Т. 44. №6. С. 29-98.

[2] С.П.Царев // Изв. АН СССР, сер. матем. 1990. Т. 54. № 5. С. 1048-1068.

[3] Е.В.Ферапонтов, С.П.Царев // Матем. моделирование. 1991. Т. 3. № 2. С. 82-91.

[4] М.В.Павлов, С.И.Свинолупов, Р.А.Шарипов // В сб.: Интегрируемость в динамических системах / Ред. Л.А.Калякин. Уфа: Институт математики, 1994. С. 30-48. (см. также solv-int@xyz.lanl.gov \#9407003).

[5] М.Б.Шефтель // Диф. уравнения. 1993. Т. 29. № 10. С. 1782-1795.

[6] М.Б.Шефтель // Диф. уравнения. 1994. Т. 30. № 3. С. 444-456.

[7] E.V.Ferapontov // Physica D. 1993. V. 63. P. 50-70.

[8] Л.П.Эйзенхарт. Риманова геометрия. М.: ИЛ, 1948.

[9] О.И.Богоявленский, С.П.Новиков // Функц. анализ и его прилож. 1976. Т. 10. С. 9-13.

[10] О.И.мохов // Изв. АН СССР, сер. матем. 1987. Т. 51. С. 1345-1352.

[11] P.Finsler. Uber Kurven und Flachen in algemeinen Raumen. Gottingen: Dissertation, 1918.

[12] E.Cartan. Les espaces de Finsler. Paris: Actualites 79, 1934.

[13] А.Ю.Болдин, В.В.Дмитриева, С.С.Сафин, Р.А.Шарипов // ТМФ. 1995. Т. 103. № 2. C. $256-266$.

[14] А.Ю.Болдин, А.А.Бронников, В.В.Дмитриева, Р.А.Шарипов // ТМФ. 1995. Т. 103. № 2. C. $267-275$.

[15] D.Serre // J. of Diff. Eq. 1983. V. 49. P. 270-280.

[16] E.V.Ferapontov // Phys. Lett. A. 1993. V. 179. P. 391-397.

[17] E.V.Ferapontov // Diff. Geometry and Appl. 1995. V. 5. P. 121-152.

[18] В.Е.Захаров, С.В.Манаков, С.П.Новиков, Л.П.Питаевский. Теория солитонов. М.: Наука, 1980.

Институт математического моделирования РАН

Поступила в редакцию

Башкирский государственный университет

17.IX.1995 г.

\section{E. V. Ferapontov, R. A. Sharipov \\ ON FIRST-ORDER CONSERVATION LAWS FOR SYSTEMS OF HYDRONAMIC TYPE EQUATIONS}

We consider conservation laws of the first order quadratic in derivatives related to systems of equations of hydrodynamic type. We derive the defining relations for their densities in a form invariant with respect to the pointwise change of variables. Examples of nondiagonalizable systems admitting quadratic conservation laws are given. 